\title{
Relaxed Extragradient Algorithms for the Split Feasibility Problem
}

\author{
Youli Yu, ${ }^{1}$ Shin Min Kang, ${ }^{2}$ and Young Chel Kwun ${ }^{3}$ \\ ${ }^{1}$ School of Mathematics and Information Engineering, Taizhou University, Linhai 317000, China \\ ${ }^{2}$ Department of Mathematics and the RINS, Gyeongsang National University, Jinju 660-701, Republic of Korea \\ ${ }^{3}$ Department of Mathematics, Dong-A University, Pusan 614-714, Republic of Korea
}

Correspondence should be addressed to Young Chel Kwun; yckwun@dau.ac.kr

Received 4 October 2013; Accepted 30 January 2014; Published 27 March 2014

Academic Editor: Li Wei

Copyright (C) 2014 Youli Yu et al. This is an open access article distributed under the Creative Commons Attribution License, which permits unrestricted use, distribution, and reproduction in any medium, provided the original work is properly cited.

The purpose of this paper is to introduce a new relaxed extragradient algorithms for the split feasibility problem. Our relaxed extragradient algorithm is new and it generalized some results for solving the split feasibility problem.

\section{Introduction}

The split feasibility problem has received much attention due to its applications in image denoising, signal processing, and image reconstruction, with particular progress in intensity modulated therapy; see, for instance [1-4]. In this paper, we continue to study the split feasibility problem and its approximation algorithms.

To begin with, let us first recall the notation of the split feasibility problem and some existing algorithms in the literature. Recall that the split feasibility problem introduced by Censor and Elfving [4] can be formulated as finding a point $x^{\dagger}$ with the property

$$
x^{\dagger} \in \mathbb{C}, \quad \mathbb{A} x^{\dagger} \in \mathbb{Q},
$$

where $\mathbb{C}$ and $\mathbb{Q}$ are two closed convex subsets of two Hilbert spaces $\mathbb{H}_{1}$ and $\mathbb{H}_{2}$, respectively, and $\mathbb{A}: \mathbb{H}_{1} \rightarrow \mathbb{H}_{2}$ is a bounded linear operator. The original algorithm introduced in [4] involves the computation of the inverse $\mathbb{A}^{-1}$ :

$$
x^{k+1}=\mathbb{A}^{-1} \operatorname{proj}_{\mathbb{Q}}\left(\operatorname{proj}_{\mathbb{A}(\mathbb{C})}\left(\mathbb{A} x^{k}\right)\right), \quad k \geq 0,
$$

where $\mathbb{C}, \mathbb{Q} \subset \mathbb{R}^{n}$ are closed convex sets and $\mathbb{A}$ is a full rank $n \times n$ matrix. It is an interesting problem to construct iterative algorithms for the split feasibility problem to the bounded linear operator.
We use $\Xi$ to denote the solution set of the split feasibility problem; that is,

$$
\Xi:=\left\{x^{\dagger} \in \mathbb{C}: \mathbb{A} x^{\dagger} \in \mathbb{Q}\right\} .
$$

In the sequel, we assume that the split feasibility problem is consistent. Let $\omega>0$ and assume that $x^{\dagger} \in \Xi$. Thus, $\mathbb{A} x^{\dagger} \in \mathbb{Q}$. It follows that $\operatorname{proj}_{\mathbb{Q}} A x^{\dagger}=\mathbb{A} x^{\dagger}$ which implies $\varpi \mathbb{A}^{*}\left(I-\operatorname{proj}_{\mathbb{Q}}\right) \mathbb{A} x^{\dagger}=0$. Hence, we deduce that the fixed point equation $\left(I-\omega \mathbb{A}^{*}\left(I-\operatorname{proj}_{\mathbb{Q}}\right) \mathbb{A}\right) x^{\dagger}=x^{\dagger}$ holds. Note that $x^{\dagger} \in \mathbb{C}$. It is obvious that

$$
\operatorname{proj}_{\mathbb{C}}\left(I-\Phi \mathbb{A}^{*}\left(I-\operatorname{proj}_{\mathbb{Q}}\right) \mathbb{A}\right) x^{\dagger}=x^{\dagger} .
$$

By using (4), Byrne [5] presented a more popular CQ algorithm:

$$
x^{k+1}=\operatorname{proj}_{\mathbb{C}}\left(x^{k}-\omega \mathbb{A}^{*}\left(I-\operatorname{proj}_{\mathbb{Q}}\right) \mathbb{A} x^{k}\right), \quad n \in \mathbb{N} .
$$

The CQ algorithm only needs to compute the projections $\operatorname{proj}_{\mathbb{C}}$ and $\operatorname{proj}_{\mathbb{Q}}$. It is therefore implementable when proj $\mathbb{C}_{\mathbb{C}}$ and proj $_{\mathbb{Q}}$ have closed-form expressions. Consequently, CQ algorithm has received so much attention; see [6-17]. In particular, in [14], Xu proposed an averaged CQ algorithm and he obtained the weak convergence result.

On the other hand, we note that $x \in \Xi$; then there exists $x^{\dagger} \in \mathbb{Q}$ such that $\mathbb{A} x-x^{\dagger}=0$. Thus, we consider the distance 
function $d\left(\mathbb{A} x, x^{\dagger}\right)=\left\|\mathbb{A} x-x^{\dagger}\right\|$ and the minimization problem

$$
\min _{x \in \mathbb{C}, x^{\dagger} \in \mathbb{Q}} \frac{1}{2}\left\|\mathbb{A} x-x^{\dagger}\right\|^{2}
$$

Since $x^{\dagger} \in \mathbb{Q}$, we then consider the minimization

$$
\min _{x \in \mathbb{C}} \varphi(x):=\frac{1}{2}\left\|\mathbb{A} x-\operatorname{proj}_{\mathbb{Q}} \mathbb{A} x\right\|^{2} .
$$

However, (7) is, in general, ill-posed. We import the wellknown Tikhonov's regularization method

$$
\min _{x \in \mathbb{C}} \varphi_{\alpha}:=\frac{1}{2}\left\|\left(I-\operatorname{proj}_{\mathbb{Q}}\right) A x\right\|^{2}+\frac{1}{2} \alpha\|x\|^{2},
$$

where $\alpha>0$ is the regularization parameter. Note that the gradient $\nabla \varphi_{\alpha}$ of $\varphi_{\alpha}$ is

$$
\nabla \varphi_{\alpha}=\nabla \varphi(x)+\alpha I=\mathbb{A}^{*}\left(I-\operatorname{proj}_{\mathbb{Q}}\right) \mathbb{A}+\alpha I .
$$

Using (9), some regularized methods have been suggested. For example, $\mathrm{Xu}$ [14] suggested the following regularized method:

$$
\begin{aligned}
x^{k+1} & =\operatorname{proj}_{\mathbb{C}}\left(I-\Theta_{k} \nabla \varphi_{\alpha_{k}}\right) x^{k} \\
& =\operatorname{proj}_{\mathbb{C}}\left(\left(1-\alpha_{k} \varrho_{k}\right) x^{k}-{\varpi_{k}} A^{*}\left(I-\operatorname{proj}_{\mathbb{Q}}\right) \mathbb{A} x^{k}\right) .
\end{aligned}
$$

He proved that the sequence $\left\{x^{k}\right\}$ generated by (10) converges to the solution of the split feasibility problem. Ceng et al. [7] presented the following extragradient method:

$$
\begin{aligned}
& x_{0}=x \in \mathbb{U}_{1} \text { chosen arbitrarily, } \\
& y^{k}=\operatorname{proj}_{\mathbb{C}}\left(x^{k}-\varrho_{k} \nabla \varphi_{\alpha_{k}}\left(x^{k}\right)\right), \\
& x^{k+1}=\beta_{k} x^{k}+\left(1-\beta_{k}\right) \operatorname{proj}_{\mathbb{C}}\left(x^{k}-\omega_{k} \nabla \varphi_{\alpha_{k}}\left(y^{k}\right)\right),
\end{aligned}
$$

$$
n \geq 0 \text {. }
$$

and proved that the sequence $\left\{x^{k}\right\}$ generated by (11) converges weakly to the solution of the split feasibility problem. Motivated by the above results, in this paper, we construct a new extragradient algorithm for solving the split feasibility problem. Strong convergence result is demonstrated.

\section{Preliminaries}

Let $\mathbb{C}$ be a nonempty closed convex subset of a real Hilbert space $\mathbb{H}$. A mapping $\Theta: \mathbb{C} \rightarrow \mathbb{C}$ is called nonexpansive if

$$
\|\Theta u-\Theta v\| \leq\|u-v\|, \quad \forall u, v \in \mathbb{C} .
$$

A mapping $W: \mathbb{C} \rightarrow \mathbb{C}$ is said to be $\nu$-inverse strong monotone, if there exists a constant $v>0$ such that

$$
\langle u-v, W u-W v\rangle \geq v\|W u-W v\|^{2}, \quad \forall u, v \in \mathbb{C} .
$$

Recall that the metric projection from $\mathbb{H}$ onto $\mathbb{C}$ denoted by $\operatorname{proj}_{\mathbb{C}}$ means that

$$
\left\|x^{\star}-\operatorname{proj}_{\mathbb{C}}\left(x^{\star}\right)\right\|=\inf \left\{\left\|x^{\star}-x\right\|: x \in \mathbb{C}\right\} .
$$

$\operatorname{proj}_{\mathbb{C}}$ can be characterized by

$$
\left\langle u-v, \operatorname{proj}_{\mathbb{C}}(u)-\operatorname{proj}_{\mathbb{C}}(v)\right\rangle \geq\left\|\operatorname{proj}_{\mathbb{C}}(u)-\operatorname{proj}_{\mathbb{C}}(v)\right\|^{2},
$$

for all $u, v \in H$. Hence, it is nonexpansive. It is also known that $2 \operatorname{proj}_{\mathbb{C}}-I$ is nonexpansive.

Let $\varphi: \mathbb{C} \rightarrow \mathbb{U}$ be a monotone mapping. The variational inequality problem is to find $x^{\dagger} \in \mathbb{C}$ such that

$$
\left\langle\varphi\left(x^{\dagger}\right), x-x^{\dagger}\right\rangle \geq 0, \quad \forall x \in \mathbb{C} .
$$

The solution set of the variational inequality is denoted by $\operatorname{VI}(\mathbb{C}, \varphi)$. It is well known that $x^{\sharp} \in \operatorname{VI}(\mathbb{C}, \varphi)$ if and only if $\mathrm{x}^{\sharp}=\operatorname{proj}_{\mathbb{C}}\left(x^{\sharp}-\lambda \varphi\left(x^{\sharp}\right)\right), \forall \lambda>0$. A set-valued mapping $R: \mathbb{U} \rightarrow 2^{\mathbb{H}}$ is called monotone if, for each $u \in \mathbb{U}, f \in R u$, and $g \in R v$, we have

$$
\langle u-v, f-g\rangle \geq 0 .
$$

A monotone mapping $R: \mathbb{H} \rightarrow 2^{\mathbb{}}$ is called maximal if its graph $G(R)$ is not properly contained in the graph of any other monotone mappings. It is known that a monotone mapping $R$ is maximal if and only if for each pair $(x, f) \in \mathbb{U} \times \mathbb{U}$ with $\langle u-v, f-g\rangle \geq 0$ for every $(v, g) \in G(R)$; then we have $f \in R u$. Let $R: \mathbb{C} \rightarrow \mathbb{W}$ be a monotone and $k$-Lipschitz continuous mapping and let $N_{\mathbb{C}} v$ be the normal cone to $\mathbb{C}$ at $v \in \mathbb{C}$; that is,

$$
N_{K} v=\{w \in \mathbb{U}:\langle v-u, w\rangle \geq 0, \forall u \in \mathbb{C}\} .
$$

Define

$$
W v= \begin{cases}R v+N_{\mathbb{C}} v, & \text { if } v \in \mathbb{C}, \\ \emptyset, & \text { if } v \notin \mathbb{C} .\end{cases}
$$

Then, $W$ is maximal monotone and $0 \in W v$ if and only if $v \in \operatorname{VI}(\mathbb{C}, R)$; see $[18]$ for more details.

Lemma 1 (see [14]). One knows the following properties:

(i) $\mathbb{A}^{*}\left(I-\operatorname{proj}_{\mathbb{Q}}\right) \mathbb{A}$ is Lipschitz continuous with Lipschitz constant $\|\mathbb{A}\|^{2}$;

(ii) $\mathbb{A}^{*}\left(I-\operatorname{proj}_{\mathbb{Q}}\right) \mathbb{A}$ is $1 /\|\mathbb{A}\|^{2}$ inverse strong monotone;

(iii) $I-\omega \mathbb{A}^{*}\left(I-\operatorname{proj}_{\mathbb{Q}}\right) A$ is nonexpansive for all $\emptyset \epsilon$ $\left(0,2 /\|\mathbb{A}\|^{2}\right)$.

Lemma 2 (see [19]). Let $\mathbb{C}$ be a nonempty closed convex subset of a real Hilbert space $\mathbb{U}$. Let the mapping $\Phi: \mathbb{C} \rightarrow \mathbb{\square}$ be $\zeta$ inverse strong monotone and let $\varrho>0$ be a constant. Then, we have

$$
\begin{gathered}
\left\|(I-\varrho \Phi) x^{\dagger}-(I-\varrho \Phi) x^{\ddagger}\right\|^{2} \\
\leq\left\|x^{\dagger}-x^{\ddagger}\right\|^{2}+\varrho(\varrho-2 \zeta)\left\|\Phi\left(x^{\dagger}\right)-\Phi\left(x^{\ddagger}\right)\right\|^{2}, \\
\forall x^{\dagger}, x^{\ddagger} \in C .
\end{gathered}
$$

In particular, if $0 \leq \varrho \leq 2 \zeta$, then $I-\varrho \Phi$ is nonexpansive. 
Lemma 3 (see [20]). Let $\left\{{ }^{k}\right\}$ and $\left\{\sigma^{k}\right\}$ be bounded sequences in a Banach space $X$ and let $\left\{\beta_{k}\right\}$ be a sequence in $[0,1]$ with $0<\liminf _{n \rightarrow \infty} \beta_{k} \leq \limsup _{n \rightarrow \infty} \beta_{k}<1$. Suppose that

$$
\iota^{k+1}=\left(1-\beta_{k}\right) \sigma^{k}+\beta_{k} \iota^{k}
$$

for all $n \geq 0$ and

$$
\limsup _{n \rightarrow \infty}\left(\left\|\sigma^{k+1}-\sigma^{k}\right\|-\left\|\iota^{k+1}-\iota^{k}\right\|\right) \leq 0 .
$$

Then, $\lim _{n \rightarrow \infty}\left\|\sigma^{k}-\iota^{k}\right\|=0$.

Lemma 4 (see [21]). Assume that $\left\{\xi_{k}\right\}$ is a sequence of nonnegative real numbers such that

$$
\xi_{k+1} \leq\left(1-\gamma_{k}\right) \xi_{k}+\vartheta_{k}
$$

where $\left\{\gamma_{k}\right\}$ is a sequence in $(0,1)$ and $\left\{\vartheta_{k}\right\}$ is a sequence such that

(1) $\sum_{n=1}^{\infty} \gamma_{k}=\infty$;

(2) $\lim \sup _{n \rightarrow \infty}\left(\vartheta_{k} / \gamma_{k}\right) \leq 0$ or $\sum_{n=1}^{\infty}\left|\vartheta_{k}\right|<\infty$.

Then, $\lim _{n \rightarrow \infty} \xi_{k}=0$.

\section{Main Results}

Let $\mathbb{W}_{1}$ and $\mathbb{\sharp}_{2}$ be two Hilbert spaces and let $\mathbb{C}$, $\mathbb{Q}$ be two nonempty closed and convex subsets of $\mathbb{H}_{1}$ and $\mathbb{H}_{2}$, respectively. Suppose that $\mathbb{A}: \mathbb{W}_{1} \rightarrow \mathbb{U}_{2}$ is a bounded linear operator and $\mathbb{F}: \mathbb{C} \rightarrow \mathbb{H}_{1}$ is a $l$-contraction. We will state and prove our main results in this paper and at the end of this paper we will give an example to show that our results improve the works in the literature.

Algorithm 5. Let $x_{0} \in \mathbb{C}$. Let $\left\{x^{k}\right\}$ be a sequence generated by

$$
\begin{array}{r}
y^{k}=\operatorname{proj}_{\mathbb{C}}\left[x^{k}-\delta_{k} \mathbb{A}^{*}\left(I-\operatorname{proj}_{\mathbb{Q}}\right) \mathbb{A} x^{k}+\alpha_{k}\left(\mathbb{F} x^{k}-x^{k}\right)\right], \\
x^{k+1}=\operatorname{proj}_{\mathbb{C}}\left[x^{k}-\lambda_{k} \mathbb{A}^{*}\left(I-\operatorname{proj}_{\mathbb{Q}}\right) \mathbb{A} y^{k}+\mu_{k}\left(y^{k}-x^{k}\right)\right], \\
n \geq \mathbb{N},
\end{array}
$$

where $\left\{\alpha_{k}\right\} \subset(0,1)$ is a sequence; $\delta_{k}, \lambda_{k} \in[a, b] \subset\left(0,2 /\|\mathbb{A}\|^{2}\right)$ and $\mu_{k} \in(0,1)$ are sequences satisfying $\lambda_{k} \leq 2 \mu_{k} /\|\mathbb{A}\|^{2}$.

Theorem 6. Suppose that $\Xi \neq \emptyset$. Assume $\lim _{n \rightarrow \infty} \alpha_{k}=0$, $\lim _{n \rightarrow \infty}\left(\lambda_{k+1}-\lambda_{k}\right)=0, \lim _{n \rightarrow \infty}\left(\delta_{k+1}-\delta_{k}\right)=0$, $\lim _{n \rightarrow \infty}\left(\mu_{k+1}-\mu_{k}\right)=0$, and $\sum_{n=1}^{\infty} \alpha_{k}=\infty$. Then the sequence $\left\{x^{k}\right\}$ generated by $(24)$ converges strongly to $\widetilde{\nu}=P_{\Xi}(\mathbb{F}) \widetilde{\nu}$.

Proof. From the assumptions $\lim _{n \rightarrow \infty} \alpha_{k}=0$ and $\delta_{k} \in$ $\left(0,2 /\|\mathbb{A}\|^{2}\right)$, we can choose $\alpha_{k}$ such that $\alpha_{k}<1-\left(\delta_{k}\|\mathbb{A}\|^{2} / 2\right)$ when $n$ is sufficiently large. For the convenience and without loss of generality, we assume that $\alpha_{k}<1-\left(\delta_{k}\|\mathbb{A}\|^{2} / 2\right)$ for all $n \in \mathbb{N}$. Then, we deduce $\delta_{k} /\left(1-\alpha_{k}\right) \in\left(0,2 /\|\mathbb{A}\|^{2}\right)$ for all $n \in \mathbb{N}$.
Let $x^{\dagger} \in \Xi$. In the first section, we know that $x^{\dagger}=$ $\operatorname{proj}_{\mathbb{C}}\left[x^{\dagger}-\xi \mathbb{A}^{*}\left(I-\operatorname{proj}_{\mathbb{Q}}\right) \mathbb{A} x^{\dagger}\right]$ for any $\xi>0$. Since $\delta_{k} /(1-$ $\left.\alpha_{k}\right) \in\left(0,2 /\|\mathbb{A}\|^{2}\right)$ and $\lambda_{k} / \mu_{k} \in\left(0,2 /\|\mathbb{A}\|^{2}\right)$, we have

$$
\begin{aligned}
x^{\dagger}=\operatorname{proj}_{\mathbb{C}} & {\left[x^{\dagger}-\frac{\delta_{k} \mathbb{A}^{*}\left(I-\operatorname{proj}_{\mathbb{Q}}\right) \mathbb{A} x^{\dagger}}{\left(1-\alpha_{k}\right)}\right] } \\
=\operatorname{proj}_{\mathbb{C}} & {\left[\alpha_{k} x^{\dagger}+\left(1-\alpha_{k}\right)\right.} \\
& \left.\times\left(x^{\dagger}-\frac{\delta_{k} \mathbb{A}^{*}\left(I-\operatorname{proj}_{\mathbb{Q}}\right) A x^{\dagger}}{\left(1-\alpha_{k}\right)}\right)\right], \quad \forall n \geq \mathbb{N}, \\
x^{\dagger}=\operatorname{proj}_{\mathbb{C}} & {\left[x^{\dagger}-\frac{\lambda_{k}}{\mu_{k} \mathbb{A}^{*}\left(I-\operatorname{proj}_{\mathbb{Q}}\right) A x^{\dagger}}\right] } \\
=\operatorname{proj}_{\mathbb{C}} & {\left[\alpha_{k} x^{\dagger}+\left(1-\alpha_{k}\right)\right.} \\
& \left.\times\left(x^{\dagger}-\frac{\lambda_{k}}{\mu_{k} \mathbb{A}^{*}\left(I-\operatorname{proj}_{\mathbb{Q}}\right) \mathbb{A} x^{\dagger}}\right)\right], \quad \forall n \geq \mathbb{N} .
\end{aligned}
$$

From (24) and (25), we have

$$
\begin{gathered}
\left\|y^{k}-x^{\dagger}\right\|=\| \operatorname{proj}_{\mathbb{C}}\left[\alpha_{k} \mathbb{F} x^{k}+\left(1-\alpha_{k}\right) x^{k}\right. \\
\left.-\delta_{k} \mathbb{A}^{*}\left(I-\operatorname{proj}_{\mathbb{Q}}\right) \mathbb{A} x^{k}\right]-x^{\dagger} \| \\
=\| \operatorname{proj}_{\mathbb{C}}\left[\alpha_{k} \mathbb{F} x^{k}+\left(1-\alpha_{k}\right)\right. \\
\left.\times\left(x^{k}-\frac{\delta_{k} \mathbb{A}^{*}\left(I-\operatorname{proj}_{\mathbb{Q}}\right) \mathbb{A} x^{k}}{\left(1-\alpha_{k}\right)}\right)\right] \\
-\operatorname{proj}_{\mathbb{C}}\left[\alpha_{k} x^{\dagger}+\left(1-\alpha_{k}\right)\right. \\
\left.\quad \times\left(x^{\dagger}-\frac{\delta_{k} \mathbb{A}^{*}\left(I-\operatorname{proj}_{\mathbb{Q}}\right) A x^{\dagger}}{\left(1-\alpha_{k}\right)}\right)\right] \| \\
\leq \mid \alpha_{k}\left(\mathbb{F} x^{k}-x^{\dagger}\right)+\left(1-\alpha_{k}\right) \\
\times\left[\left(x^{k}-\frac{\delta_{k} \mathbb{A}^{*}\left(I-\operatorname{proj}_{\mathbb{Q}}\right) \mathbb{A} x^{k}}{\left(1-\alpha_{k}\right)}\right)\right. \\
\left.-\left(x^{\dagger}-\frac{\delta_{k} \mathbb{A}^{*}\left(I-\operatorname{proj}_{\mathbb{Q}}\right) \mathbb{A} x^{\dagger}}{\left(1-\alpha_{k}\right)}\right)\right] \| .
\end{gathered}
$$


From Lemma 1, we know that $I-\delta_{k} \mathbb{A}^{*}\left(I-\operatorname{proj}_{\mathbb{Q}}\right) \mathbb{A} /\left(1-\alpha_{k}\right)$ and $I-\lambda_{k} / \mu_{k} \mathbb{A}^{*}\left(I-\operatorname{proj}_{\mathbb{Q}}\right) \mathbb{A}$ are nonexpansive. It follows that

$$
\begin{gathered}
\left\|y^{k}-x^{\dagger}\right\| \\
\leq\left(1-\alpha_{k}\right) \|\left(I-\frac{\delta_{k}}{\left(1-\alpha_{k}\right) \mathbb{A}^{*}\left(I-\operatorname{proj}_{\mathbb{Q}}\right) \mathbb{A}}\right) x^{k} \\
-\left(I-\frac{\delta_{k}}{\left(1-\alpha_{k}\right) \mathbb{A}^{*}\left(I-\operatorname{proj}_{\mathbb{Q}}\right) \mathbb{A}}\right) x^{\dagger} \| \\
+\alpha_{k}\left\|\mathbb{F} x^{k}-\mathbb{F} x^{\dagger}\right\|+\alpha_{k}\left\|\mathbb{F} x^{\dagger}-x^{\dagger}\right\| \\
\leq \alpha_{k} \iota\left\|x^{k}-x^{\dagger}\right\|+\alpha_{k}\left\|\mathbb{F} x^{\dagger}-x^{\dagger}\right\|+\left(1-\alpha_{k}\right)\left\|x^{k}-x^{\dagger}\right\| \\
=\left[1-(1-\imath) \alpha_{k}\right]\left\|x^{k}-x^{\dagger}\right\|+\alpha_{k}\left\|\mathbb{F} x^{\dagger}-x^{\dagger}\right\| .
\end{gathered}
$$

Thus,

$$
\begin{aligned}
& \left\|x^{k+1}-x^{\dagger}\right\| \\
& =\| \operatorname{proj}_{\mathbb{C}}\left[x^{k}-\lambda_{k} \mathbb{A}^{*}\left(I-\operatorname{proj}_{\mathbb{Q}}\right) \mathbb{A} y^{k}\right. \\
& \left.+\mu_{k}\left(y^{k}-x^{k}\right)\right]-x^{\dagger} \| \\
& =\| \operatorname{proj}_{\mathbb{C}}\left[\left(1-\mu_{k}\right) x^{k}\right. \\
& \left.+\mu_{k}\left(y^{k}-\frac{\lambda_{k}}{\mu_{k} \mathbb{A}^{*}\left(I-\operatorname{proj}_{\mathbb{Q}}\right) \mathbb{A} y^{k}}\right)\right] \\
& -\operatorname{proj}_{\mathbb{C}}\left[\left(1-\mu_{k}\right) x^{\dagger}\right. \\
& \left.+\mu_{k}\left(x^{\dagger}-\frac{\lambda_{k}}{\mu_{k} \mathbb{A}^{*}\left(I-\operatorname{proj}_{\mathbb{Q}}\right) A x^{\dagger}}\right)\right] \| \\
& \leq\left(1-\mu_{k}\right)\left\|x^{k}-x^{\dagger}\right\| \\
& +\mu_{k} \|\left(y^{k}-\frac{\lambda_{k}}{\mu_{k} \mathbb{A}^{*}\left(I-\operatorname{proj}_{\mathbb{Q}}\right) \mathbb{A} y^{k}}\right) \\
& -\left(x^{\dagger}-\frac{\lambda_{k}}{\mu_{k} \mathbb{A}^{*}\left(I-\operatorname{proj}_{\mathbb{Q}}\right) \mathbb{A} x^{\dagger}}\right) \| \\
& \leq\left(1-\mu_{k}\right)\left\|x^{k}-x^{\dagger}\right\|+\mu_{k}\left\|y^{k}-x^{\dagger}\right\| \\
& \leq\left(1-\mu_{k}\right)\left\|x^{k}-x^{\dagger}\right\|+\mu_{k} \alpha_{k}\left\|\mathbb{F} x^{\dagger}-x^{\dagger}\right\| \\
& +\mu_{k}\left[1-(1-\imath) \alpha_{k}\right]\left\|x^{k}-x^{\dagger}\right\| \\
& =\left(1-(1-\iota) \mu_{k} \alpha_{k}\right)\left\|x^{k}-x^{\dagger}\right\|+\mu_{k} \alpha_{k}\left\|\mathbb{F} x^{\dagger}-x^{\dagger}\right\| \\
& \leq \max \left\{\frac{\left\|\mathbb{F} x^{\dagger}-x^{\dagger}\right\|}{1-\iota},\left\|x_{0}-x^{\dagger}\right\|\right\} \text {. }
\end{aligned}
$$

Hence, $\left\{x^{k}\right\}$ is bounded. It follows from (28) that the sequence $\left\{y^{k}\right\}$ is also bounded.
Set $\mathbb{S}=2 \operatorname{proj}_{\mathbb{C}}-I$. Note that $S$ is nonexpansive. Thus, we can rewrite $x^{k+1}$ in (24) as

$$
\begin{aligned}
x^{k+1} & \\
= & \frac{I+\mathbb{S}}{2}\left[\left(1-\mu_{k}\right) x^{k}+\mu_{k}\left(y^{k}-\frac{\lambda_{k}}{\mu_{k} \mathbb{A}^{*}\left(I-\operatorname{proj}_{\mathbb{Q}}\right) \mathbb{A} y^{k}}\right)\right] \\
= & \frac{1-\mu_{k}}{2} x^{k}+\frac{\mu_{k}}{2}\left(y^{k}-\frac{\lambda_{k}}{\mu_{k} \mathbb{A}^{*}\left(I-\operatorname{proj}_{\mathbb{Q}}\right) \mathbb{A} y^{k}}\right) \\
& +\frac{1}{2} \mathbb{S}\left(\left(1-\mu_{k}\right) x^{k}+\mu_{k}\left(y^{k}-\frac{\lambda_{k}}{\mu_{k} \mathbb{A}^{*}\left(I-\operatorname{proj}_{\mathbb{Q}}\right) \mathbb{A} y^{k}}\right)\right) \\
= & \frac{1-\mu_{k}}{2} x^{k}+\frac{1+\mu_{k}}{2} z^{k},
\end{aligned}
$$

where

$$
\begin{aligned}
& z^{k} \\
& =\left[\mu_{k}\left(y^{k}-\frac{\lambda_{k}}{\mu_{k} \mathbb{A}^{*}\left(I-\operatorname{proj}_{\mathbb{Q}}\right) \mathbb{A} y^{k}}\right)\right. \\
& \left.\quad+\mathbb{S}\left(\left(1-\mu_{k}\right) x^{k}+\mu_{k}\left(y^{k}-\frac{\lambda_{k}}{\mu_{k} \mathbb{A}^{*}\left(I-\operatorname{proj}_{\mathbb{Q}}\right) \mathbb{A} y^{k}}\right)\right)\right] \\
& \quad \times\left(1+\mu_{k}\right)^{-1} .
\end{aligned}
$$

It follows that

$$
\begin{aligned}
& z^{k+1}-z^{k} \\
& =\mu_{k+1}\left[\left(y^{k+1}-\frac{\lambda_{k+1}}{\mu_{k+1} \mathbb{A}^{*}\left(I-\operatorname{proj}_{\mathbb{Q}}\right) \mathbb{A} y^{k+1}}\right)\right. \\
& +\mathbb{S}\left(\left(1-\mu_{k+1}\right) x^{k+1}+\mu_{k+1}\right. \\
& \left.\left.\quad \times\left(y^{k+1}-\frac{\lambda_{k+1}}{\mu_{k+1} \mathbb{A}^{*}\left(I-\operatorname{proj}_{\mathbb{Q}}\right) \mathbb{A} y^{k+1}}\right)\right)\right] \\
& \times\left(1+\mu_{k+1}\right)^{-1} \\
& -\mu_{k}\left[\left(y^{k}-\frac{\lambda_{k}}{\mu_{k} A^{*}\left(I-\operatorname{proj}_{\mathbb{Q}}\right) A y^{k}}\right)\right. \\
& +\mathbb{S}\left(\left(1-\mu_{k}\right) x^{k}\right. \\
& \left.\left.\quad+\mu_{k}\left(y^{k}-\frac{\lambda_{k}}{\mu_{k} \mathbb{A}^{*}\left(I-\operatorname{proj}_{\mathbb{Q}}\right) \mathbb{A} y^{k}}\right)\right)\right] \\
& \times\left(1+\mu_{k}\right)^{-1} .
\end{aligned}
$$


Journal of Applied Mathematics

5

So,

$$
\left\|z^{k+1}-z^{k}\right\|
$$

$\leq \frac{\mu_{k+1}}{1+\mu_{k+1}} \|\left(y^{k+1}-\frac{\lambda_{k+1}}{\mu_{k+1} A^{*}\left(I-\operatorname{proj}_{\mathbb{Q}}\right) A y^{k+1}}\right)$

$$
-\left(y^{k}-\frac{\lambda_{k}}{\mu_{k} \mathbb{A}^{*}\left(I-\operatorname{proj}_{\mathbb{Q}}\right) \mathbb{A} y^{k}}\right) \|
$$

$+\left|\frac{\mu_{k+1}}{1+\mu_{k+1}}-\frac{u^{k}}{1+\mu_{k}}\right|$

$$
\begin{aligned}
& \times \| y^{k}-\frac{\lambda_{k}}{\mu_{k} \mathbb{A}^{*}\left(I-\operatorname{proj}_{\mathbb{Q}}\right) \mathbb{A} y^{k}} \|+\frac{1}{1+\mu_{k+1}} \\
& \times \| \mathbb{S}\left[\left(1-\mu_{k+1}\right) x^{k+1}+\mu_{k+1}\right. \\
&\left.\quad \times\left(y^{k+1}-\frac{\lambda_{k+1}}{\mu_{k+1} \mathbb{A}^{*}\left(I-\operatorname{proj}_{\mathbb{Q}}\right) \mathbb{A} y^{k+1}}\right)\right]
\end{aligned}
$$

$$
\begin{aligned}
-S( & \left(1-\mu_{k}\right) x^{k}+\mu_{k} \\
& \left.\times\left(y^{k}-\frac{\lambda_{k}}{\mu_{k} \mathbb{A}^{*}\left(I-\operatorname{proj}_{\mathbb{Q}}\right) \mathbb{A} y^{k}}\right)\right) \|
\end{aligned}
$$

$$
\begin{aligned}
& +\left|\frac{1}{1+\mu_{k+1}}-\frac{1}{1+\mu_{k}}\right| \\
& \times \| \mathbb{S}\left(\left(1-\mu_{k}\right) x^{k}\right. \\
& \left.\quad+\mu_{k}\left(y^{k}-\frac{\lambda_{k}}{\mu_{k} \mathbb{A}^{*}\left(I-\operatorname{proj}_{\mathbb{Q}}\right) \mathbb{A} y^{k}}\right)\right) \|
\end{aligned}
$$

$$
\begin{aligned}
\leq \frac{\mu_{k+1}}{1+\mu_{k+1}} \| & \left(I-\frac{\lambda_{k+1}}{\mu_{k+1} A^{*}\left(I-\operatorname{proj}_{\mathbb{Q}}\right) \mathbb{A}}\right) y^{k+1} \\
& -\left(I-\frac{\lambda_{k+1}}{\mu_{k+1} \mathbb{A}^{*}\left(I-\operatorname{proj}_{\mathbb{Q}}\right) \mathbb{A}}\right) y^{k} \|
\end{aligned}
$$

$+\frac{\mu_{k+1}}{1+\mu_{k+1}}\left|\frac{\lambda_{k+1}}{\mu_{k+1}}-\frac{\lambda_{k}}{\mu_{k}}\right|\left\|\mathbb{A} y^{k}\right\|+\left|\frac{\mu_{k+1}}{1+\mu_{k+1}}-\frac{u^{k}}{1+\mu_{k}}\right|$

$\times\left\|y^{k}-\frac{\lambda_{k}}{\mu_{k} \mathbb{A}^{*}\left(I-\operatorname{proj}_{\mathbb{Q}}\right) \mathbb{A} y^{k}}\right\|$

$+\frac{1}{1+\mu_{k+1}} \|\left(1-\mu_{k+1}\right) x^{k+1}+\mu_{k+1}$

$$
\begin{aligned}
& \times\left(y^{k+1}-\frac{\lambda_{k+1}}{\mu_{k+1} \mathbb{A}^{*}\left(I-\operatorname{proj}_{\mathbb{Q}}\right) \mathbb{A} y^{k+1}}\right) \\
& -\left(1-\mu_{k}\right) x^{k} \\
& -\mu_{k}\left(y^{k}-\frac{\lambda_{k}}{\mu_{k} \mathbb{A}^{*}\left(I-\operatorname{proj}_{\mathbb{Q}}\right) \mathbb{A} y^{k}}\right) \|
\end{aligned}
$$

$$
\begin{aligned}
& +\left|\frac{1}{1+\mu_{k+1}}-\frac{1}{1+\mu_{k}}\right| \\
& \times \| \mathbb{S}\left(\left(1-\mu_{k}\right) x^{k}\right. \\
& \left.\quad+\mu_{k}\left(y^{k}-\frac{\lambda_{k}}{\mu_{k} \mathbb{A}^{*}\left(I-\operatorname{proj}_{\mathbb{Q}}\right) \mathbb{A} y^{k}}\right)\right) \| .
\end{aligned}
$$

It follows that

$$
\begin{aligned}
& \left\|z^{k+1}-z^{k}\right\| \\
& \leq \frac{\mu_{k+1}}{1+\mu_{k+1}}\left\|y^{k+1}-y^{k}\right\|+\frac{\mu_{k+1}}{1+\mu_{k+1}}\left|\frac{\lambda_{k+1}}{\mu_{k+1}}-\frac{\lambda_{k}}{\mu_{k}}\right|\left\|\mathrm{A} y^{k}\right\| \\
& +\left|\frac{\mu_{k+1}}{1+\mu_{k+1}}-\frac{u^{k}}{1+\mu_{k}}\right| \\
& \times\left\|y^{k}-\frac{\lambda_{k}}{\mu_{k}} \mathbb{A}^{*}\left(I-\operatorname{proj}_{\mathbb{Q}}\right) \mathbb{A} y^{k}\right\|+\frac{1}{1+\mu_{k+1}} \\
& \times \|\left(1-\mu_{k+1}\right)\left(x^{k+1}-x^{k}\right)+\left(\mu_{k}-\mu_{k+1}\right)\left(x^{k}-y^{k}\right) \\
& +\mu_{k+1}\left[\left(I-\frac{\lambda_{k+1}}{\mu_{k+1} \mathbb{A}^{*}\left(I-\operatorname{proj}_{\mathbb{Q}}\right) \mathbb{A}}\right) y^{k+1}\right. \\
& \left.-\left(I-\frac{\lambda_{k+1}}{\mu_{k+1} \mathbb{A}^{*}\left(I-\operatorname{proj}_{\mathbb{Q}}\right) \mathbb{A}}\right) y^{k}\right] \\
& +\left(\lambda_{k}-\lambda_{k+1}\right) \mathbb{A}^{*}\left(I-\operatorname{proj}_{\mathbb{Q}}\right) \mathbb{A} y^{k} \\
& +\left|\frac{1}{1+\mu_{k+1}}-\frac{1}{1+\mu_{k}}\right| \\
& \times \| \mathbb{S}\left(\left(1-\mu_{k}\right) x^{k}\right. \\
& \left.+\mu_{k}\left(y^{k}-\frac{\lambda_{k}}{\mu_{k} \mathbb{A}^{*}\left(I-\operatorname{proj}_{\mathbb{Q}}\right) \mathbb{A} y^{k}}\right)\right) \| \\
& \leq \frac{2 \mu_{k+1}}{1+\mu_{k+1}}\left\|y^{k+1}-y^{k}\right\|+\frac{1-\mu_{k+1}}{1+\mu_{k+1}}\left\|x^{k+1}-x^{k}\right\| \\
& +\frac{\mu_{k+1}}{1+\mu_{k+1}}\left|\frac{\lambda_{k+1}}{\mu_{k+1}}-\frac{\lambda_{k}}{\mu_{k}}\right|\left\|\mathbb{A} y^{k}\right\| \\
& +\frac{\left|\mu_{k}-\mu_{k+1}\right|}{1+\mu_{k+1}}\left\|x^{k}-y^{k}\right\|+\left|\frac{\mu_{k+1}}{1+\mu_{k+1}}-\frac{u^{k}}{1+\mu_{k}}\right| \\
& \times\left\|y^{k}-\frac{\lambda_{k}}{\mu_{k} \mathbb{A}^{*}\left(I-\operatorname{proj}_{\mathbb{Q}}\right) \mathbb{A} y^{k}}\right\| \\
& +\left|\frac{1}{1+\mu_{k+1}}-\frac{1}{1+\mu_{k}}\right|
\end{aligned}
$$


6

Journal of Applied Mathematics

$$
\begin{aligned}
& \times \| \mathbb{S}\left(\left(1-\mu_{k}\right) x^{k}\right. \\
& \left.\quad+\mu_{k}\left(y^{k}-\frac{\lambda_{k}}{\mu_{k} \mathbb{A}^{*}\left(I-\operatorname{proj}_{\mathbb{Q}}\right) \mathbb{A} y^{k}}\right)\right) \| \\
& +\frac{\left|\lambda_{k}-\lambda_{k+1}\right|}{1+\mu_{k+1}}\left\|\mathbb{A}^{*}\left(I-\operatorname{proj}_{\mathbb{Q}}\right) \mathbb{A} y^{k}\right\| .
\end{aligned}
$$

From (24), we have

$$
\begin{aligned}
& \left\|y^{k+1}-y^{k}\right\| \\
& =\| \operatorname{proj}_{\mathbb{C}}\left[\alpha_{k+1} \mathbb{F} x^{k+1}+\left(1-\alpha_{k+1}\right) x^{k+1}\right. \\
& \left.-\delta_{k+1} \mathbb{A}^{*}\left(I-\operatorname{proj}_{\mathbb{Q}}\right) \mathbb{A} x^{k+1}\right] \\
& -\operatorname{proj}_{\mathbb{C}}\left[\alpha_{k} \mathbb{F} x^{k}+\left(1-\alpha_{k}\right) x^{k}\right. \\
& \left.-\delta_{k} \mathbb{A}^{*}\left(I-\operatorname{proj}_{\mathbb{Q}}\right) \mathbb{A} x^{k}\right] \| \\
& \leq \|\left[\alpha_{k+1} \mathbb{F} x^{k+1}+\left(1-\alpha_{k+1}\right) x^{k+1}\right. \\
& \left.-\delta_{k+1} \mathbb{A}^{*}\left(I-\operatorname{proj}_{\mathbb{Q}}\right) \mathbb{A} x^{k+1}\right] \\
& -\left[\alpha_{k} \mathbb{F} x^{k}+\left(1-\alpha_{k}\right) x^{k}-\delta_{k} \mathbb{A}^{*}\left(I-\operatorname{proj}_{\mathbb{Q}}\right) \mathbb{A} x^{k}\right] \| \\
& \leq \|\left(I-\delta_{k+1} \mathbb{A}^{*}\left(I-\operatorname{proj}_{\mathbb{Q}}\right) \mathbb{A}\right) x^{k+1} \\
& -\left(I-\delta_{k+1} \mathbb{A}^{*}\left(I-\operatorname{proj}_{\mathbb{Q}}\right) \mathbb{A}\right) x^{k} \| \\
& +\alpha_{k+1}\left\|\mathbb{F} x^{k+1}-x^{k+1}\right\|+\left|\delta_{k+1}-\delta_{k}\right| \\
& \times\left\|\mathbb{A}^{*}\left(I-\operatorname{proj}_{\mathbb{Q}}\right) \mathbb{A} x^{k}\right\|+\alpha_{k}\left\|\mathbb{F} x^{k}-x^{k}\right\| \\
& \leq\left\|x^{k+1}-x^{k}\right\|+\left|\delta_{k+1}-\delta_{k}\right|\left\|\mathbb{A}^{*}\left(I-\operatorname{proj}_{\mathbb{Q}}\right) \mathbb{A} x^{k}\right\| \\
& +\alpha_{k+1}\left\|\mathbb{F} x^{k+1}-x^{k+1}\right\|+\alpha_{k}\left\|\mathbb{F} x^{k}-x^{k}\right\| \text {. }
\end{aligned}
$$

Hence, we deduce

$$
\begin{aligned}
& \left\|z^{k+1}-z^{k}\right\| \\
& \leq\left\|x^{k+1}-x^{k}\right\|+\left|\delta_{k+1}-\delta_{k}\right|\left\|\mathbb{A}^{*}\left(I-\operatorname{proj}_{\mathbb{Q}}\right) \mathbb{A} x^{k}\right\| \\
& \quad+\alpha_{k+1}\left\|\mathbb{F} x^{k+1}-x^{k+1}\right\|+\alpha_{k}\left\|\mathbb{F} x^{k}-x^{k}\right\| \\
& \quad+\frac{\mu_{k+1}}{1+\mu_{k+1}}\left|\frac{\lambda_{k+1}}{\mu_{k+1}}-\frac{\lambda_{k}}{\mu_{k}}\right|\left\|\mathbb{A} y^{k}\right\| \\
& \quad+\left|\frac{\mu_{k+1}}{1+\mu_{k+1}}-\frac{u^{k}}{1+\mu_{k}}\right|\left\|y^{k}-\frac{\lambda_{k}}{\mu_{k} \mathbb{A}^{*}\left(I-\operatorname{proj}_{\mathbb{Q}}\right) \mathbb{A} y^{k}}\right\|
\end{aligned}
$$

$$
\begin{aligned}
& +\frac{\left|\mu_{k}-\mu_{k+1}\right|}{1+\mu_{k+1}}\left\|x^{k}-y^{k}\right\|+\left|\frac{1}{1+\mu_{k+1}}-\frac{1}{1+\mu_{k}}\right| \\
& \times\left\|\mathbb{S}\left(\left(1-\mu_{k}\right) x^{k}+\mu_{k}\left(y^{k}-\frac{\lambda_{k}}{\mu_{k} \mathbb{A}^{*}\left(I-\operatorname{proj}_{\mathbb{Q}}\right) \mathbb{A} y^{k}}\right)\right)\right\| \\
& +\frac{\left|\lambda_{k}-\lambda_{k+1}\right|}{1+\mu_{k+1}}\left\|\mathbb{A}^{*}\left(I-\operatorname{proj}_{\mathbb{Q}}\right) \mathbb{A} y^{k}\right\| .
\end{aligned}
$$

Since $\lim _{n \rightarrow \infty} \alpha_{k}=0, \lim _{n \rightarrow \infty}\left(\lambda_{k+1}-\lambda_{k}\right)=0$, $\lim _{n \rightarrow \infty}\left(\delta_{k+1}-\delta_{k}\right)=0$, and $\lim _{n \rightarrow \infty}\left(\mu_{k+1}-\mu_{k}\right)=0$, we derive that

$$
\limsup _{n \rightarrow \infty}\left(\left\|z^{k+1}-z^{k}\right\|-\left\|x^{k+1}-x^{k}\right\|\right) \leq 0 .
$$

By Lemma 3, we obtain

$$
\lim _{n \rightarrow \infty}\left\|z^{k}-x^{k}\right\|=0 .
$$

Hence,

$$
\lim _{n \rightarrow \infty}\left\|x^{k+1}-x^{k}\right\|=\lim _{n \rightarrow \infty} \frac{1+\mu_{k}}{2}\left\|z^{k}-x^{k}\right\|=0 .
$$

By Lemma 1, Lemma 2, and (29), we get

$$
\begin{aligned}
\| x^{k+1}- & x^{\dagger} \|^{2} \\
\leq & \left(1-\mu_{k}\right)\left\|x^{k}-x^{\dagger}\right\|^{2}+\mu_{k}\left\|y^{k}-x^{\dagger}\right\|^{2} \\
\leq & \left(1-\mu_{k}\right)\left\|x^{k}-x^{\dagger}\right\|^{2} \\
+ & \mu_{k} \| \alpha_{k}\left(\mathbb{F} x^{k}-x^{\dagger}\right)+\left(1-\alpha_{k}\right) \\
& \times\left[\left(x^{k}-\frac{\delta_{k}}{\left(1-\alpha_{k}\right) \mathbb{A}^{*}\left(I-\operatorname{proj}_{\mathbb{Q}}\right) \mathbb{A} x^{k}}\right)\right. \\
\leq & \left.-\left(x^{\dagger}-\frac{\delta_{k}}{\left(1-\alpha_{k}\right) \mathbb{A}^{*}\left(I-\operatorname{proj}_{\mathbb{Q}}\right) \mathbb{A} x^{\dagger}}\right)\right] \|^{2} \\
& {\left[\alpha_{k}\left\|\mathbb{F} x^{k}-x^{\dagger}\right\|^{2}+\left(1-\alpha_{k}\right)\right.} \\
& \times \|\left(I-\frac{\delta_{k}}{\left(1-\alpha_{k}\right) \mathbb{A}^{*}\left(I-\operatorname{proj}_{\mathbb{Q}}\right) \mathbb{A}}\right) x^{k} \\
& \left.\left(I-\frac{\delta_{k}}{\left(1-\alpha_{k}\right) \mathbb{A}^{*}\left(I-\operatorname{proj}_{\mathbb{Q}}\right) \mathbb{A}}\right) x^{\dagger} \|^{2}\right]
\end{aligned}
$$




$$
\begin{aligned}
& +\left(1-\mu_{k}\right)\left\|x^{k}-x^{\dagger}\right\|^{2} \\
\leq & \mu_{k} \delta_{k}\left(\frac{\delta_{k}}{1-\alpha_{k}}-\frac{2}{\|\mathbb{A}\|^{2}}\right) \\
& \times\left\|\mathbb{A}^{*}\left(I-\operatorname{proj}_{\mathbb{Q}}\right) \mathbb{A} x^{k}-\mathbb{A}^{*}\left(I-\operatorname{proj}_{\mathbb{Q}}\right) \mathbb{A} x^{\dagger}\right\|^{2} \\
& +\left(1-\alpha_{k}\right) \mu_{k}\left\|x^{k}-x^{\dagger}\right\|^{2} \\
& +\left(1-\mu_{k}\right)\left\|x^{k}-x^{\dagger}\right\|^{2}+\alpha_{k} \mu_{k}\left\|\mathbb{F} x^{k}-x^{\dagger}\right\|^{2} \\
\leq & \mu_{k} a\left(\frac{b}{1-\alpha_{k}}-\frac{2}{\|\mathbb{A}\|^{2}}\right) \\
& \times\left\|\mathbb{A}^{*}\left(I-\operatorname{proj}_{\mathbb{Q}}\right) \mathbb{A} x^{k}-\mathbb{A}^{*}\left(I-\operatorname{proj}_{\mathbb{Q}}\right) \mathbb{A} x^{\dagger}\right\|^{2} \\
& +\alpha_{k}\left\|\mathbb{F} x^{k}-x^{\dagger}\right\|^{2}+\left\|x^{k}-x^{\dagger}\right\|^{2} .
\end{aligned}
$$

Therefore, we have

$$
\begin{aligned}
\mu_{k} a & \left(\frac{2}{\|\mathbb{A}\|^{2}}-\frac{b}{\left(1-\alpha_{k}\right)}\right) \\
& \times\left\|\mathbb{A}^{*}\left(I-\operatorname{proj}_{\mathbb{Q}}\right) \mathbb{A} x^{k}-A^{*}\left(I-\operatorname{proj}_{\mathbb{Q}}\right) \mathbb{A} x^{\dagger}\right\|^{2} \\
\leq & \alpha_{k}\left\|\mathbb{F} x^{k}-x^{\dagger}\right\|^{2}+\left\|x^{k}-x^{\dagger}\right\|^{2}-\left\|x^{k+1}-x^{\dagger}\right\|^{2} \\
\leq & \alpha_{k}\left\|\mathbb{F} x^{k}-x^{\dagger}\right\|^{2}+\left(\left\|x_{n}-x^{\dagger}\right\|+\left\|x^{k+1}-x^{\dagger}\right\|\right) \\
& \times\left\|x^{k}-x^{k+1}\right\| .
\end{aligned}
$$

Noting that $0<a<\lambda_{k}<2 \mu_{k} /\|\mathbb{A}\|^{2}$, we get $\liminf _{n \rightarrow \infty} \mu_{k}>$ 0 . Since $\lim _{n \rightarrow \infty} \alpha_{k}=0$ and $b<2 /\|\mathbb{A}\|^{2}$, we obtain $\liminf _{n \rightarrow \infty} \mu_{k} a\left(2 /\|\mathbb{A}\|^{2}-b /\left(1-\alpha_{k}\right)\right)>0$. Thus, we have

$$
\lim _{n \rightarrow \infty}\left\|\mathbb{A}^{*}\left(I-\operatorname{proj}_{\mathbb{Q}}\right) A x^{k}-\mathbb{A}^{*}\left(I-\operatorname{proj}_{\mathbb{Q}}\right) A x^{\dagger}\right\|=0 .
$$

Using (15), we have

$$
\left\|y^{k}-x^{\dagger}\right\|^{2}
$$$$
=\| \operatorname{proj}_{\mathbb{C}}\left[\alpha_{k} \mathbb{F} x^{k}+\left(1-\alpha_{k}\right) x^{k}-\delta_{k} \mathbb{A}^{*}\left(I-\operatorname{proj}_{\mathbb{Q}}\right) \mathbb{A} x^{k}\right]
$$$$
-\operatorname{proj}_{\mathbb{C}}\left[x^{\dagger}-\delta_{k} \mathbb{A}^{*}\left(I-\operatorname{proj}_{\mathbb{Q}}\right) A x^{\dagger}\right] \|^{2}
$$$$
\leq\left\langle\alpha_{k} \mathbb{F} x^{k}+\left(1-\alpha_{k}\right) x^{k}-\delta_{k} \mathbb{A}^{*}\left(I-\operatorname{proj}_{\mathbb{Q}}\right) \mathbb{A} x^{k}\right.
$$$$
\left.-\left(x^{\dagger}-\delta_{k} \mathbb{A}^{*}\left(I-\operatorname{proj}_{\mathbb{Q}}\right) \mathbb{A} x^{\dagger}\right), y^{k}-x^{\dagger}\right\rangle
$$

$$
\begin{aligned}
&=\frac{1}{2}\{\| x^{k}-\delta_{k} \mathbb{A}^{*}\left(I-\operatorname{proj}_{\mathbb{Q}}\right) \mathbb{A} x^{k} \\
&-\left(x^{\dagger}-\delta_{k} \mathbb{A}^{*}\left(I-\operatorname{proj}_{\mathbb{Q}}\right) \mathbb{A} x^{\dagger}\right) \\
&+ \alpha_{k}\left(\mathbb{F} x^{k}-x^{k}\right)\left\|^{2}+\right\| y^{k}-x^{\dagger} \|^{2} \\
&- \| \alpha_{k} \mathbb{F} x^{k}+\left(1-\alpha_{k}\right) x^{k}-\delta_{k} \mathbb{A}^{*}\left(I-\operatorname{proj}_{\mathbb{Q}}\right) \mathbb{A} x^{k} \\
&\left.-\left(x^{\dagger}-\delta_{k} \mathbb{A}^{*}\left(I-\operatorname{proj}_{\mathbb{Q}}\right) \mathbb{A} x^{\dagger}\right)-\left(y^{k}-x^{\dagger}\right) \|^{2}\right\} \\
& \leq \frac{1}{2}\left\{\|\left(x^{k}-\delta_{k} \mathbb{A}^{*}\left(I-\operatorname{proj}_{\mathbb{Q}}\right) \mathbb{A} x^{k}\right)\right. \\
&-\left(x^{\dagger}-\delta_{k} \mathbb{A}^{*}\left(I-\operatorname{proj}_{\mathbb{Q}}\right) \mathbb{A} x^{\dagger}\right) \|^{2} \\
&+2 \alpha_{k}\left\|\mathbb{F} x^{k}-x^{k}\right\| \\
& \times \| x^{k}-\delta_{k} \mathbb{A}^{*}\left(I-\operatorname{proj}_{\mathbb{Q}}\right) \mathbb{A} x^{k} \\
& \quad-\left(x^{\dagger}-\delta_{k} \mathbb{A}^{*}\left(I-\operatorname{proj}_{\mathbb{Q}}\right) \mathbb{A} x^{\dagger}\right) \\
&\left.+\alpha_{k}\left(\mathbb{F} x^{k}-x^{k}\right) \|^{2}\right\} \\
&+\alpha_{k}\left(\mathbb{F} x^{k}-x^{k}\right)\|+\| y^{k}-x^{\dagger} \|^{2} \\
&-\|\left(x^{k}-y^{k}\right) \\
&-
\end{aligned}
$$

$$
\begin{aligned}
\leq \frac{1}{2}\left\{\left\|x^{k}-x^{\dagger}\right\|^{2}+\alpha_{k} M+\left\|y^{k}-x^{\dagger}\right\|^{2}\right. \\
-\left\|x^{k}-y^{k}\right\|^{2}+2 \alpha_{k}\left\langle x^{k}, x^{k}-y^{k}\right\rangle \\
+2 \delta_{k}\left\langle x^{k}-y^{k}, \mathbb{A}^{*}\left(I-\operatorname{proj}_{\mathbb{Q}}\right) \mathbb{A} x^{k}\right. \\
\left.-\mathbb{A}^{*}\left(I-\operatorname{proj}_{\mathbb{Q}}\right) \mathbb{A} x^{\dagger}\right\rangle \\
-\| \delta_{k}\left(\mathbb{A}^{*}\left(I-\operatorname{proj}_{\mathbb{Q}}\right) \mathbb{A} x^{k}-\mathbb{A}^{*}\left(I-\operatorname{proj}_{\mathbb{Q}}\right) \mathbb{A} x^{\dagger}\right) \\
\left.-\alpha_{k}\left(\mathbb{F} x^{k}-x^{k}\right) \|^{2}\right\}
\end{aligned}
$$




$$
\begin{aligned}
\leq \frac{1}{2}\{ & \left\|x^{k}-x^{\dagger}\right\|^{2}+\alpha_{k} M+\left\|y^{k}-x^{\dagger}\right\|^{2} \\
& -\left\|x^{k}-y^{k}\right\|^{2}+2 \alpha_{k}\left\|\mathbb{F} x^{k}-x^{k}\right\|\left\|x^{k}-y^{k}\right\| \\
& +2 \delta_{k}\left\|x^{k}-y^{k}\right\| \\
& \left.\times\left\|\mathbb{A}^{*}\left(I-\operatorname{proj}_{\mathbb{Q}}\right) \mathbb{A} x^{k}-\mathbb{A}^{*}\left(I-\operatorname{proj}_{\mathbb{Q}}\right) \mathbb{A} x^{\dagger}\right\|\right\},
\end{aligned}
$$

where $M>0$ is some constant such that

$$
\begin{gathered}
\sup _{k}\left\{2\left\|x^{k}\right\| \| x^{k}-\delta_{k} \mathbb{A}^{*}\left(I-\operatorname{proj}_{\mathbb{Q}}\right) \mathbb{A} x^{k}\right. \\
-\left(x^{\dagger}-\delta_{k} \mathbb{A}^{*}\left(I-\operatorname{proj}_{\mathbb{Q}}\right) \mathbb{A} x^{\dagger}\right) \\
\left.+\alpha_{k}\left(\mathbb{F} x^{k}-x^{k}\right) \|\right\} \leq M .
\end{gathered}
$$

It follows that

$$
\begin{aligned}
\left\|y^{k}-x^{\dagger}\right\|^{2} \leq & \left\|x^{k}-x^{\dagger}\right\|^{2}+\alpha_{k} M-\left\|x^{k}-y^{k}\right\|^{2} \\
& +2 \alpha_{k}\left\|\mathbb{F} x^{k}-x^{k}\right\|\left\|x^{k}-y^{k}\right\|+2 \delta_{k}\left\|x^{k}-y^{k}\right\| \\
& \times\left\|\mathbb{A}^{*}\left(I-\operatorname{proj}_{\mathbb{Q}}\right) \mathbb{A} x^{k}-\mathbb{A}^{*}\left(I-\operatorname{proj}_{\mathbb{Q}}\right) \mathbb{A} x^{\dagger}\right\|,
\end{aligned}
$$

and hence

$$
\begin{aligned}
\| x^{k+1}- & x^{\dagger} \|^{2} \\
\leq & \left(1-\mu_{k}\right)\left\|x^{k}-x^{\dagger}\right\|^{2}+\mu_{k}\left\|y^{k}-x^{\dagger}\right\|^{2} \\
\leq & \left\|x^{k}-x^{\dagger}\right\|^{2}+\alpha_{k} M-\mu_{k}\left\|x^{k}-y^{k}\right\|^{2} \\
& +2 \alpha_{k}\left\|\mathbb{F} x^{k}-x^{k}\right\|\left\|x^{k}-y^{k}\right\|+2 \delta_{k}\left\|x^{k}-y^{k}\right\| \\
& \times\left\|\mathbb{A}^{*}\left(I-\operatorname{proj}_{\mathbb{Q}}\right) \mathbb{A} x^{k}-\mathbb{A}^{*}\left(I-\operatorname{proj}_{\mathbb{Q}}\right) \mathbb{A} x^{\dagger}\right\|
\end{aligned}
$$

which implies that

$$
\begin{aligned}
\mu_{k} \| x^{k}- & y^{k} \|^{2} \\
\leq & \left(\left\|x^{k}-x^{\dagger}\right\|+\left\|x^{k+1}-x^{\dagger}\right\|\right)\left\|x^{k+1}-x^{k}\right\| \\
& +\alpha_{k} M+2 \alpha_{k}\left\|\mathbb{F} x^{k}-x^{k}\right\|\left\|x^{k}-y^{k}\right\|+2 \delta_{k}\left\|x^{k}-y^{k}\right\| \\
& \times\left\|\mathbb{A}^{*}\left(I-\operatorname{proj}_{\mathbb{Q}}\right) \mathbb{A} x^{k}-\mathbb{A}^{*}\left(I-\operatorname{proj}_{\mathbb{Q}}\right) \mathbb{A} x^{\dagger}\right\| .
\end{aligned}
$$

Since $\alpha_{k} \rightarrow 0,\left\|x_{n}-x^{k+1}\right\| \rightarrow 0$ and $\| \mathbb{A}^{*}\left(I-\operatorname{proj}_{\mathbb{Q}}\right) \mathbb{A} x^{k}-$ $\mathbb{A}^{*}\left(I-\operatorname{proj}_{\mathbb{Q}}\right) \mathbb{A} x^{\dagger} \| \rightarrow 0$, we derive

$$
\lim _{n \rightarrow \infty}\left\|x^{k}-y^{k}\right\|=0 \text {. }
$$

Next we show that

$$
\limsup _{n \rightarrow \infty}\left\langle\widetilde{v}-\mathbb{F} \widetilde{v}, \widetilde{v}-y^{k}\right\rangle \leq 0
$$

where $\widetilde{v}=P_{\Xi}(\mathbb{F} \widetilde{v})$. To show it, we choose a subsequence $\left\{y_{n_{i}}\right\}$ of $\left\{y^{k}\right\}$ such that

$$
\limsup _{n \rightarrow \infty}\left\langle\widetilde{v}-\mathbb{F} \widetilde{v}, \widetilde{v}-y^{k}\right\rangle=\lim _{i \rightarrow \infty}\left\langle\widetilde{v}-\mathbb{F} \widetilde{\mathcal{v}}, \widetilde{v}-y_{n_{i}}\right\rangle
$$

Since $\left\{y^{k}\right\}$ is bounded and $\left\{y_{n_{i}}\right\}$ is then bounded, we have the fact that a subsequence $\left\{y_{n_{i j}}\right\}$ of $\left\{y_{n_{i}}\right\}$ converges weakly to $z$.

Next we show that $z \in \Xi$. We define a mapping $W$ by

$$
W v= \begin{cases}\mathbb{A}^{*}\left(I-\operatorname{proj}_{\mathbb{Q}}\right) \mathbb{A} v+N_{\mathbb{C}} v, & v \in \mathbb{C}, \\ \emptyset, & v \notin \mathbb{C} .\end{cases}
$$

Then $W$ is maximal monotone. Let $(v, w) \in G(W)$. Since $w-$ $\mathbb{A}^{*}\left(I-\operatorname{proj}_{\mathbb{Q}}\right) \mathbb{A} v \in N_{\mathbb{C}} v$ and $y^{k} \in \mathbb{C}$, we get

$$
\left\langle v-y^{k}, w-\mathbb{A}^{*}\left(I-\operatorname{proj}_{\mathbb{Q}}\right) \mathbb{A} v\right\rangle \geq 0
$$

On the other hand, from $y^{k}=\operatorname{proj}_{\mathbb{C}}\left[\alpha_{k} \mathbb{F} x^{k}+\left(1-\alpha_{k}\right) x^{k}-\right.$ $\left.\delta_{k} \mathbb{A}^{*}\left(I-\operatorname{proj}_{\mathbb{Q}}\right) \mathbb{A} x^{k}\right]$, we have

$$
\begin{array}{r}
\left\langle v-y^{k}, y^{k}-\alpha_{k} \mathbb{F} x^{k}-\left(1-\alpha_{k}\right) x^{k}\right. \\
\left.+\delta_{k} \mathbb{A}^{*}\left(I-\operatorname{proj}_{\mathbb{Q}}\right) \mathbb{A} x^{k}\right\rangle \geq 0 .
\end{array}
$$

That is,

$$
\begin{aligned}
& \left\langle v-y^{k}, \frac{\left(y^{k}-x^{k}\right)}{\delta_{k}}+\mathbb{A}^{*}\left(I-\operatorname{proj}_{\mathbb{Q}}\right) \mathbb{A} x^{k}\right. \\
& \left.+\frac{\alpha_{k}}{\delta_{k}\left(x^{k}-\mathbb{F} x^{k}\right)}\right\rangle \geq 0 .
\end{aligned}
$$

Therefore, we have

$$
\begin{aligned}
& \left\langle v-y_{n_{i}}, w\right\rangle \\
& \geq\left\langle v-y_{n_{i}}, \mathbb{A} v\right\rangle \geq\left\langle v-y_{n_{i}}, \mathbb{A}^{*}\left(I-\operatorname{proj}_{\mathbb{Q}}\right) \mathbb{A} v\right\rangle \\
& -\left\langle v-y_{n_{i}}, \frac{\left(y_{n_{i}}-x_{n_{i}}\right)}{\delta_{n_{i}}}+\mathbb{A}^{*}\left(I-\operatorname{proj}_{\mathbb{Q}}\right) \mathbb{A} x_{n_{i}}\right. \\
& \left.+\frac{\alpha_{n_{i}}}{\delta_{n_{i}}(I-\mathbb{F}) x_{n_{i}}}\right\rangle \\
& =\left\langle v-y_{n_{i}}, \mathbb{A}^{*}\left(I-\operatorname{proj}_{\mathbb{Q}}\right) \mathbb{A} v\right. \\
& -\mathbb{A}^{*}\left(I-\operatorname{proj}_{\mathbb{Q}}\right) \mathbb{A} x_{n_{i}}-\frac{\left(y_{n_{i}}-x_{n_{i}}\right)}{\delta_{n_{i}}} \\
& \left.-\frac{\alpha_{n_{i}}}{\delta_{n_{i}}(I-\mathbb{F}) x_{n_{i}}}\right\rangle
\end{aligned}
$$




$$
\begin{aligned}
= & \left\langle v-y_{n_{i}}, \mathbb{A}^{*}\left(I-\operatorname{proj}_{\mathbb{Q}}\right) \mathbb{A} v-\mathbb{A}^{*}\left(I-\operatorname{proj}_{\mathbb{Q}}\right) \mathbb{A} y_{n_{i}}\right\rangle \\
& -\left\langle v-y_{n_{i}}, \frac{\left(y_{n_{i}}-x_{n_{i}}\right)}{\delta_{n_{i}}}+\frac{\alpha_{n_{i}}}{\delta_{n_{i}}(I-\mathbb{F}) x_{n_{i}}}\right\rangle \\
& +\left\langle v-y_{n_{i}}, \mathbb{A}^{*}\left(I-\operatorname{proj}_{\mathbb{Q}}\right) \mathbb{A} y_{n_{i}}\right. \\
& \left.-\mathbb{A}^{*}\left(I-\operatorname{proj}_{\mathbb{Q}}\right) \mathbb{A} x_{n_{i}}\right\rangle \\
\geq & \left\langle v-y_{n_{i}}, \mathbb{A}^{*}\left(I-\operatorname{proj}_{\mathbb{Q}}\right) \mathbb{A} y_{n_{i}}-\mathbb{A}^{*}\left(I-\operatorname{proj}_{\mathbb{Q}}\right) \mathbb{A} x_{n_{i}}\right\rangle \\
& -\left\langle v-y_{n_{i}}, \frac{\left(y_{n_{i}}-x_{n_{i}}\right)}{\delta_{n_{i}}}+\frac{\alpha_{n_{i}}}{\delta_{n_{i}}(I-\mathbb{F}) x_{n_{i}}}\right\rangle .
\end{aligned}
$$

Noting that $\alpha_{n_{i}} \rightarrow 0,\left\|y_{n_{i}}-x_{n_{i}}\right\| \rightarrow 0$ and $\mathbb{A}^{*}\left(I-\operatorname{proj}_{\mathbb{Q}}\right) \mathbb{A}$ is Lipschitz continuous, we deduce from above

$$
\langle v-z, w\rangle \geq 0 .
$$

Since $W$ is maximal monotone, we have $z \in W^{-1}(0)$ and hence

$$
z \in \operatorname{VI}\left(\mathbb{C}, \mathbb{A}^{*}\left(I-\operatorname{proj}_{\mathbb{Q}}\right) \mathbb{A}\right)=\Xi
$$

Therefore,

$$
\begin{aligned}
& \limsup _{n \rightarrow \infty}\left\langle\widetilde{v}-\mathbb{F} \widetilde{\nu}, \widetilde{\nu}-y^{k}\right\rangle \\
& \quad=\lim _{i \rightarrow \infty}\left\langle\widetilde{v}-\mathbb{F} \widetilde{\nu}, \widetilde{v}-y_{n_{i}}\right\rangle=\langle\widetilde{v}-\mathbb{F} \widetilde{\nu}, \widetilde{v}-z\rangle \leq 0 .
\end{aligned}
$$

Again from (15), we have

$$
\begin{aligned}
& \left\|y^{k}-\widetilde{v}\right\|^{2} \\
& =\| \operatorname{proj}_{\mathbb{C}}\left[\alpha_{k} \mathbb{F} x^{k}+\left(1-\alpha_{k}\right)\right. \\
& \left.\times\left(x^{k}-\frac{\delta_{k}}{\left(1-\alpha_{k}\right) \mathbb{A}^{*}\left(I-\operatorname{proj}_{\mathbb{Q}}\right) \mathbb{A} x^{k}}\right)\right] \\
& -\operatorname{proj}_{\mathbb{C}}\left[\alpha_{k} \widetilde{v}+\left(1-\alpha_{k}\right)\right. \\
& \left.\times\left(\tilde{\nu}-\frac{\delta_{k}}{\left(1-\alpha_{k}\right) A^{*}\left(I-\operatorname{proj}_{\mathbb{Q}}\right) A \widetilde{\nu}}\right)\right] \|^{2} \\
& \leq\left\langle\alpha_{k}\left(\mathbb{F} x^{k}-\widetilde{v}\right)+\left(1-\alpha_{k}\right)\right. \\
& \times\left[\left(x^{k}-\frac{\delta_{k}}{\left(1-\alpha_{k}\right) \mathbb{A}^{*}\left(I-\operatorname{proj}_{\mathbb{Q}}\right) \mathbb{A} x^{k}}\right)\right. \\
& \left.\left.-\left(\tilde{v}-\frac{\delta_{k}}{\left(1-\alpha_{k}\right) \mathbb{A}^{*}\left(I-\operatorname{proj}_{\mathbb{Q}}\right) A \widetilde{v}}\right)\right], y^{k}-\tilde{v}\right\rangle
\end{aligned}
$$

$$
\begin{aligned}
& \leq \alpha_{k}\left\langle\widetilde{\nu}-\mathbb{F} x^{k}, \widetilde{\nu}-y^{k}\right\rangle+\left(1-\alpha_{k}\right) \\
& \times \|\left(x^{k}-\frac{\delta_{k}}{\left(1-\alpha_{k}\right) A^{*}\left(I-\operatorname{proj}_{\mathbb{Q}}\right) A x^{k}}\right) \\
& -\left(\widetilde{\nu}-\frac{\delta_{k}}{\left(1-\alpha_{k}\right) \mathbb{A}^{*}\left(I-\operatorname{proj}_{\mathbb{Q}}\right) \mathbb{A} \widetilde{\nu}}\right)\|\| y^{k}-\widetilde{\nu} \| \\
& \leq \alpha_{k}\left\langle\widetilde{v}-\mathbb{F} \widetilde{\nu}, \widetilde{v}-y^{k}\right\rangle+\alpha_{k}\left\langle\mathbb{F} \widetilde{v}-\mathbb{F} x^{k}, \widetilde{v}-y^{k}\right\rangle \\
& +\left(1-\alpha_{k}\right)\left\|x^{k}-\tilde{\nu}\right\|\left\|y^{k}-\tilde{\nu}\right\| \\
& \leq \alpha_{k}\left\langle\widetilde{\nu}-\mathbb{F} \widetilde{\nu}, \widetilde{\nu}-y^{k}\right\rangle+\alpha_{k}\left\|\mathbb{F} \widetilde{\nu}-\mathbb{F} x^{k}\right\| \\
& \times\left\|\tilde{\nu}-y^{k}\right\|+\left(1-\alpha_{k}\right)\left\|x^{k}-\tilde{\nu}\right\|\left\|y^{k}-\tilde{v}\right\| \\
& \leq \alpha_{k}\left\langle\widetilde{v}-\mathbb{F} \widetilde{\nu}, \widetilde{v}-y^{k}\right\rangle+\left[1-(1-\iota) \alpha_{k}\right]\left\|x^{k}-\widetilde{\nu}\right\|\left\|y^{k}-\widetilde{\nu}\right\| \\
& \leq \alpha_{k}\left\langle\tilde{v}-\mathbb{F} \widetilde{\nu}, \tilde{v}-y^{k}\right\rangle+\frac{1-(1-\imath) \alpha_{k}}{2}\left\|x^{k}-\widetilde{\nu}\right\|^{2} \\
& +\frac{1}{2}\left\|y^{k}-\tilde{v}\right\|^{2} \text {. }
\end{aligned}
$$

Hence,

$$
\begin{aligned}
\left\|y^{k}-\widetilde{\nu}\right\|^{2} \leq & {\left[1-(1-\imath) \alpha_{k}\right]\left\|x^{k}-\widetilde{\nu}\right\|^{2} } \\
& +2 \alpha_{k}\left\langle\widetilde{v}-\mathbb{F} \widetilde{v}, \widetilde{v}-y^{k}\right\rangle .
\end{aligned}
$$

Therefore,

$$
\begin{aligned}
\left\|x^{k+1}-\tilde{\nu}\right\|^{2} \leq & \left(1-\mu_{k}\right)\left\|x^{k}-\tilde{\nu}\right\|^{2}+\mu_{k}\left\|y^{k}-\widetilde{\nu}\right\|^{2} \\
\leq & {\left[1-\mu_{k}(1-\imath) \alpha_{k}\right]\left\|x^{k}-\widetilde{\nu}\right\|^{2} } \\
& +2 \mu_{k} \alpha_{k}\left\langle\widetilde{\nu}-\mathbb{F} \widetilde{\nu}, \widetilde{\nu}-y^{k}\right\rangle .
\end{aligned}
$$

We apply Lemma 4 to the last inequality to deduce that $x^{k} \rightarrow$ $\widetilde{v}$. This completes the proof.

\section{Conflict of Interests}

The authors declare that there is no conflict of interests regarding the publication of this paper.

\section{Acknowledgments}

The authors would like to thank the referees for the useful comments and suggestions. This study was supported by research funds from Dong-A University.

\section{References}

[1] H. H. Bauschke and J. M. Borwein, "On projection algorithms for solving convex feasibility problems," SIAM Review, vol. 38, no. 3, pp. 367-426, 1996. 
[2] C. Byrne, "A unified treatment of some iterative algorithms in signal processing and image reconstruction," Inverse Problems, vol. 20, no. 1, pp. 103-120, 2004.

[3] Y. Censor, T. Bortfeld, B. Martin, and A. Trofimov, "A unified approach for inversion problems in intensity modulated radiation therapy," Physics in Medicine and Biology, vol. 51, pp. 23532365, 2006.

[4] Y. Censor and T. Elfving, "A multiprojection algorithm using Bregman projections in a product space," Numerical Algorithms, vol. 8, no. 2-4, pp. 221-239, 1994.

[5] C. Byrne, "Iterative oblique projection onto convex sets and the split feasibility problem," Inverse Problems, vol. 18, no. 2, pp. 441453, 2002.

[6] C. Byrne, Y. Censor, A. Gibali, and S. Reich, "Weak and strong convergence of algorithms for the split common null point problem," Journal of Nonlinear and Convex Analysis, vol. 13, no. 4, pp. 759-775, 2012.

[7] L.-C. Ceng, Q. H. Ansari, and J.-C. Yao, "An extragradient method for solving split feasibility and fixed point problems," Computers \& Mathematics with Applications, vol. 64, no. 4, pp. 633-642, 2012.

[8] Y. Dang and Y. Gao, "The strong convergence of a KM-CQ-like algorithm for a split feasibility problem," Inverse Problems, vol. 27, no. 1, Article ID 015007, 9 pages, 2011.

[9] A. Moudafi, "A relaxed alternating CQ-algorithm for convex feasibility problems," Nonlinear Analysis: Theory, Methods \& Applications, vol. 79, pp. 117-121, 2013.

[10] A. Moudafi, "Alternating CQ-algorithm for convex feasibility and splitfixed-point problems," Journal of Nonlinear and Convex Analysis. In press.

[11] M. A. Noor and K. I. Noor, "Some new classes of quasi split feasibility problems," Applied Mathematics \& Information Sciences, vol. 7, no. 4, pp. 1547-1552, 2013.

[12] B. Qu and N. Xiu, "A note on the CQ algorithm for the split feasibility problem," Inverse Problems, vol. 21, no. 5, pp. 1655$1665,2005$.

[13] H.-K. Xu, "A variable Krasnosel'skii-Mann algorithm and the multiple-set split feasibility problem," Inverse Problems, vol. 22, no. 6, pp. 2021-2034, 2006.

[14] H.-K. Xu, "Iterative methods for the split feasibility problem in infinite-dimensional Hilbert spaces," Inverse Problems, vol. 26, no. 10, Article ID 105018, 17 pages, 2010.

[15] J. Zhao and Q. Yang, "Several solution methods for the split feasibility problem," Inverse Problems, vol. 21, no. 5, pp. 17911799, 2005.

[16] Y. Yao, T. H. Kim, S. Chebbi, and H. K. Xu, "A modified extragradient method for the split feasibility and fixed point problems," Journal of Nonlinear and Convex Analysis, vol. 13, pp. 383-396, 2012.

[17] Y. Yao, W. Jigang, and Y.-C. Liou, "Regularized methods for the split feasibility problem," Abstract and Applied Analysis, vol. 2012, Article ID 140679, 13 pages, 2012.

[18] R. T. Rockafellar, "On the maximality of sums of nonlinear monotone operators," Transactions of the American Mathematical Society, vol. 149, pp. 75-88, 1970.

[19] W. Takahashi and M. Toyoda, "Weak convergence theorems for nonexpansive mappings and monotone mappings," Journal of Optimization Theory and Applications, vol. 118, no. 2, pp. 417428, 2003.

[20] T. Suzuki, "Strong convergence theorems for infinite families of nonexpansive mappings in general Banach spaces," Fixed Point Theory and Applications, vol. 2005, no. 1, pp. 103-123, 2005.
[21] H.-K. Xu, "Iterative algorithms for nonlinear operators," Journal of the London Mathematical Society, vol. 66, no. 1, pp. 240-256, 2002. 


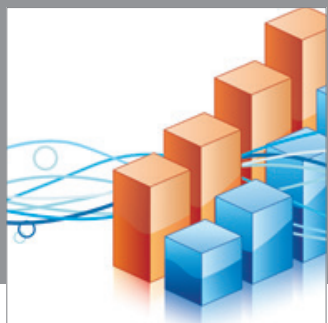

Advances in

Operations Research

mansans

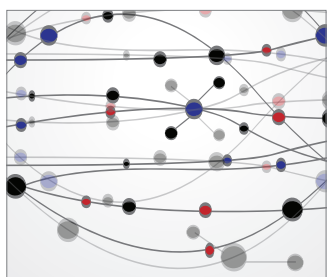

The Scientific World Journal
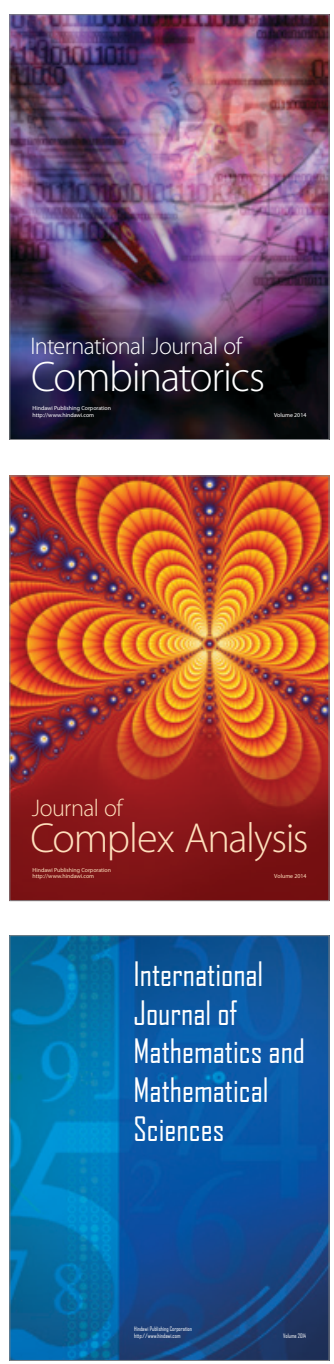
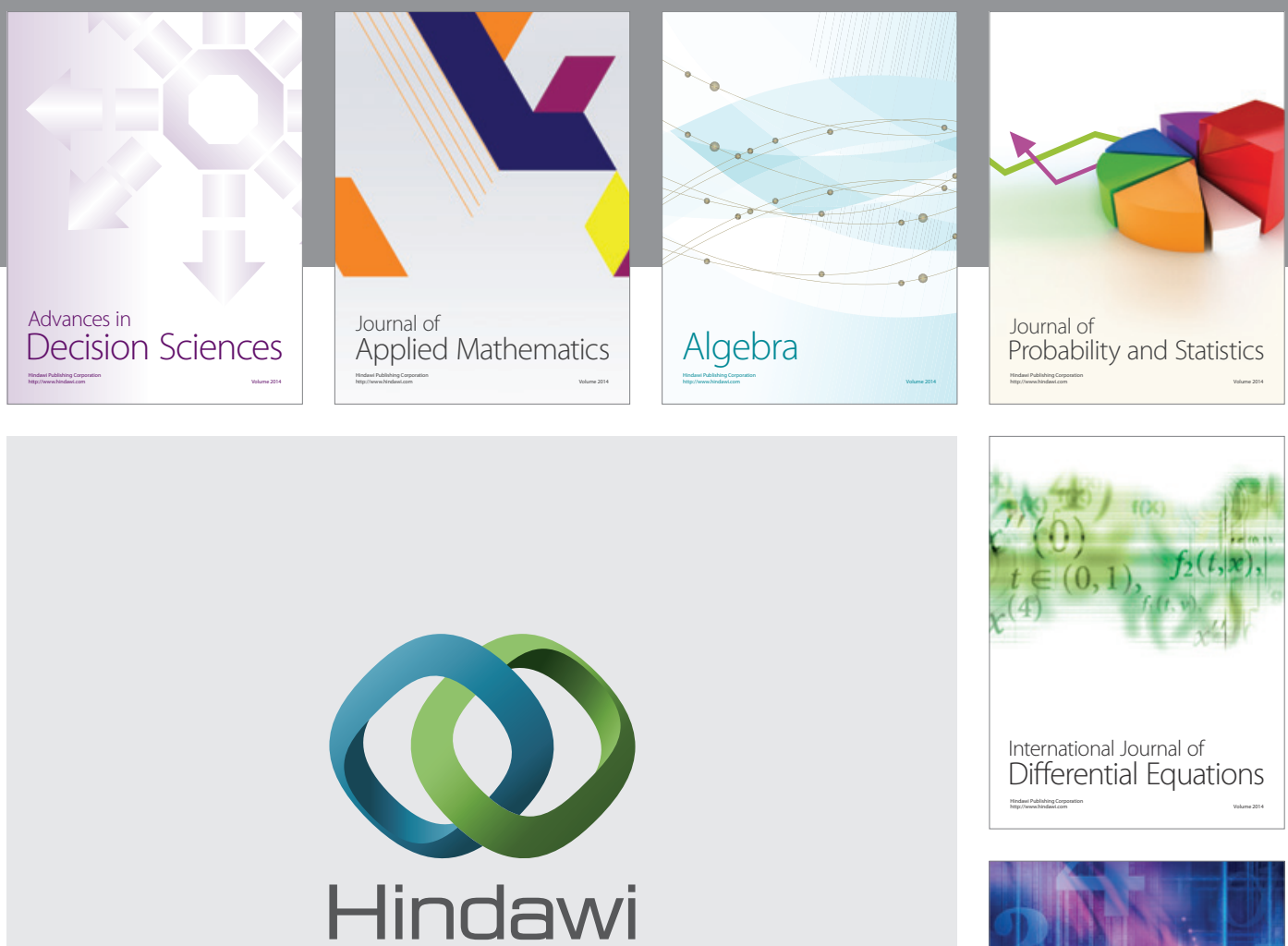

Submit your manuscripts at http://www.hindawi.com
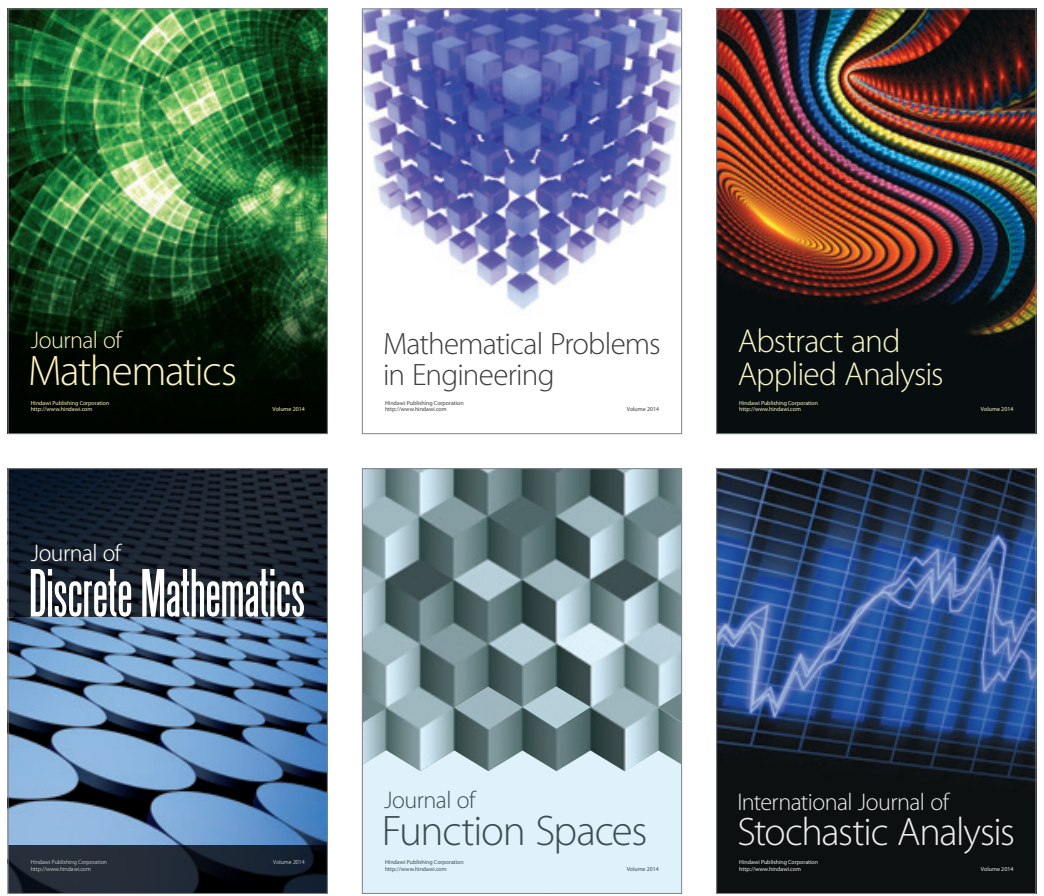

Journal of

Function Spaces

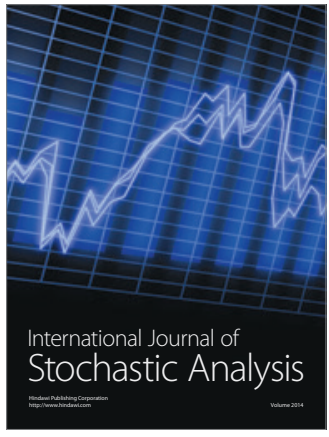

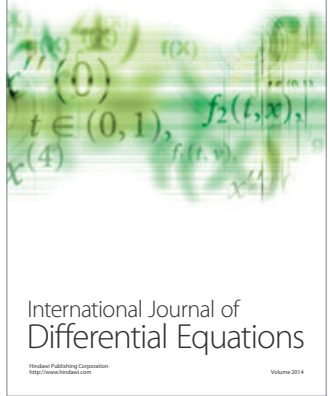
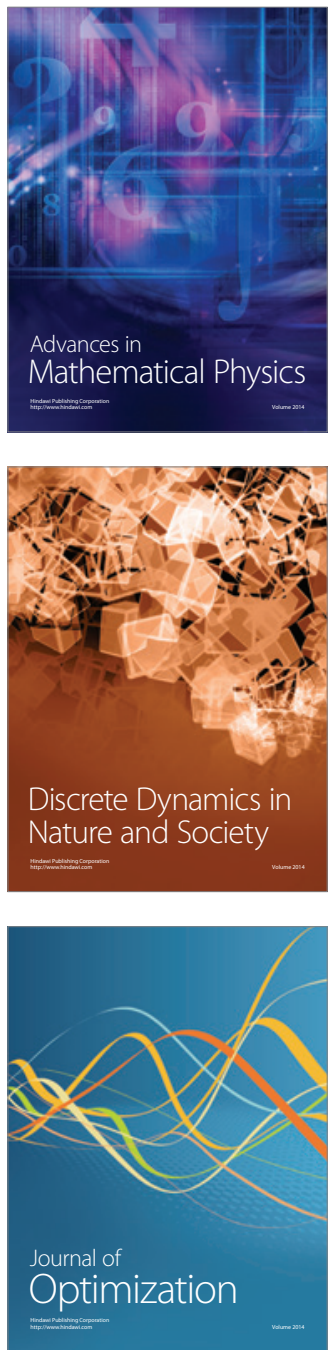